\title{
Atrofia muscular espinal en tratamiento: reporte de un caso
}

\section{Spinal muscular atrophy in treatment: case report}

\author{
Sergio Birnbaun ${ }^{1,2}$, Julieta Mozzoni ${ }^{3}$
}

\section{RESUMEN}

La atrofia muscular espinal (AME) es una enfermedad neuromuscular de la infancia, que se transmite en forma autosómica recesiva. Se conocen diferentes formas clínicas, según la edad de inicio de los síntomas y la capacidad de marcha.

Recientemente, se ha desarrollado una medicación que involucra la modulación de una proteína codificada por el gen relacionado con la sobrevida de las neuronas motoras, demostrando eficiencia en la mejoría de la función muscular. Presentamos un caso de un niño de 6 años con AME, en tratamiento que ha demostrado una significativa mejora de su capacidad motora.

Palabras clave: atrofia muscular espinal; motoneuronas; tratamiento.

\section{ABSTRACT}

Spinal muscular atrophy (SMA) is a neuromuscular disease of childhood. It is transmitted in an autosomal recessive manner. Different clinical forms are known, according to the age of onset of symptoms and the ability to walk. Recently, a medicine has been developed that targets the modulation of a protein encoded by the gene related to the survival of motor neurons, demonstrating efficiency in the improvement of muscle function.

We present a case of a 6 year old boy with SMA, who has shown a significant improvement in his motor ability with this a treatment.

Keywords: spinal muscular atrophy; motor neurons; treatment.

Fronteras en Medicina 20 I 8; |3(3): I73- 175. Doi: I 0.3 | 954/RFEM/20 I803/0 I73-0 I75

\section{INTRODUCCIÓN}

La atrofia muscular espinal (AME) es una enfermedad caracterizada por atrofia y debilidad muscular. Más del 95\% de los casos se asocian a un trastorno autosómico recesivo por una deleción o mutación homocigótica en el gen 5q13 que regula la expresión de una proteína que contribuye a la supervivencia de la neurona motora $(\mathrm{SMN1})^{1}$.

La incidencia de esta enfermedad es aproximadamente de $1 / 10.000$ nacidos vivos, con una frecuencia portadora de $1 / 54^{1,2}$.

La severidad del AME es variable y se relaciona con un gen secundario, denominado SMN2. Este gen, también produce una fracción mensajero de la misma proteína producida por el gen $S M N 1$. Se ha demostrado que existe una correlación entre el número de copias del gen $S M N 2$ y la gravedad clínica ${ }^{3,4}$.

El abordaje terapéutico de esta enfermedad más recien-

I. Instituto del Niño, Mendoza.

2. Centro Médico Palmares, Godoy Cruz, Mendoza.

3. Hospital Garraham, CABA.

Correspondencia: Dr. Sergio Birnbaun. Instituto del Niño. Av. San Martín 14I8, 550I Mendoza,Argentina. Tel: 026I 424-5364. Email: sergiobirbaun@gmail.com

Los autores declaran no poseer conflictos de intereses.

Recibido: 15/06/2018 |Aceptado: 22/7/2018 te involucra la modulación de los niveles de la proteína codificada por el gen $S M N 2^{5}$, demostrando eficiencia en la mejoría de la función muscular ${ }^{6,7}$.

Presentamos el caso de un niño de 5 años con diagnóstico de AME (II) que ha comenzado con tratamiento biológico, completando a la fecha 6 aplicaciones.

\section{CASO CLÍNICO}

El niño nació sin antecedentes patológicos prenatales a las 39 semanas de embarazo. Su desarrollo motriz fue normal los primeros meses con adecuada pos-

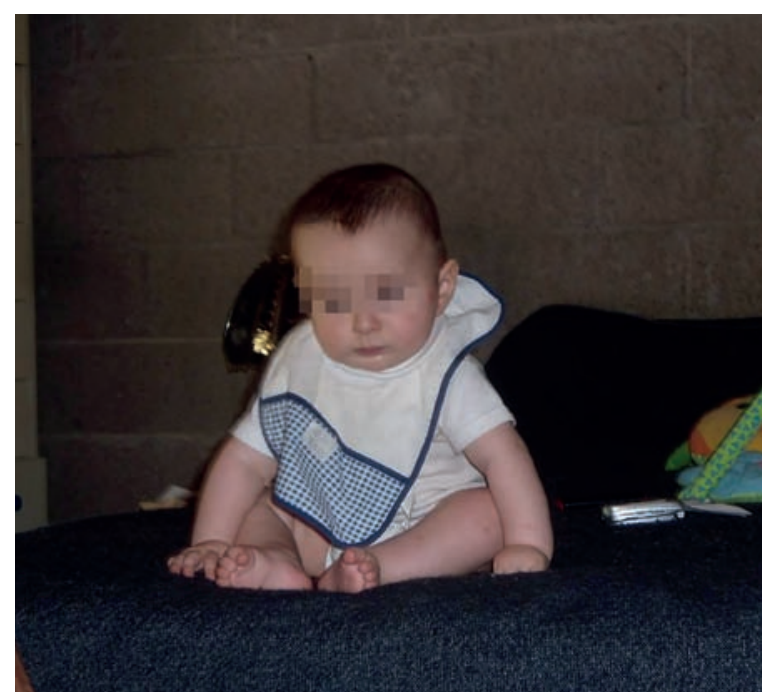

Figura I. Imagen del niño a los 7 meses en posición sentado. 


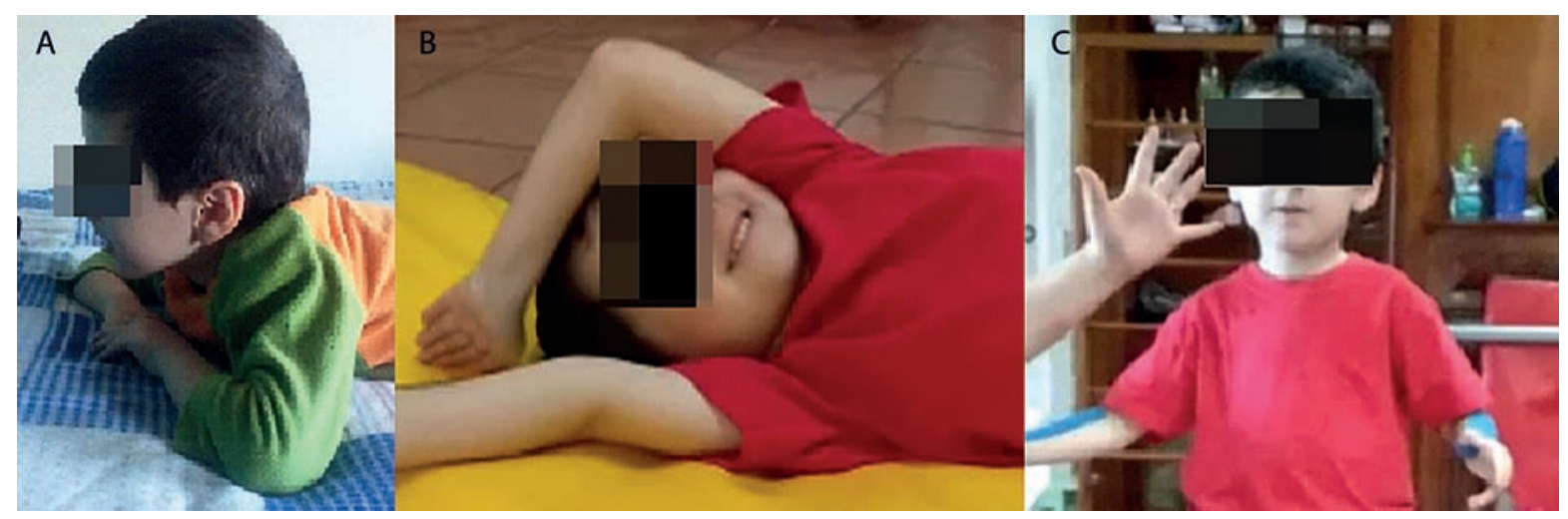

Figura 2. A) Mantuvo la postura prono con mejor sostén de la cabeza. B) Comenzó a rolar. C) Postura erguida con buen sostén.

tura para sentarse alrededor de los 6 meses (Figura 1), aunque la debilidad muscular, acentuándose temporalmente, impedía que el niño pudiera rolar, gatear o parase. Al cumplir el primer año, se evidenció una hipotonía generalizada a predominio distal de los cuatro miembros y reflejos osteotendinosos (rot) negativos que derivaron en una consulta neurológica. A la edad de 16 meses, se decidió realizar un electromiograma con estudio de conducción nerviosa motora y latencias sensitivas de los cuatro miembros, que resultó en un patrón neurofisiológico compatible con polirradiconeuropatía mixta con compromiso axonal predominante, sin compromiso sensitivo. Análisis bioquímicos demostraron valores de carnitina en sangre dentro de parámetros normales (libre $16.7 \mu \mathrm{M} / \mathrm{l}$; esterificada $18.9 \mu \mathrm{M} / 1$ : 10.4 y total $35.7 \mu \mathrm{M} / \mathrm{l})$. Posteriormente, el estudio molecular determinó que el niño presenta una deleción homocigota de ambos exones 7 y 8 del gen SMN1.

La edad de inicio de los síntomas clínicos, sumado al diagnóstico molecular confirmó la sospecha diagnóstica de AME tipo II. Se comenzó tratamiento kinésico e hidroterapia. Se mantuvo estable, sin alteraciones en la deglución, evaluación cardiológica y laboratorio normal hasta los 4 años, excepto por una fractura de cadera que tuvo buena evolución. Se decidió comenzar con 3 $\mathrm{mg}$ de salbutamol/día que luego se incrementó a 6 por vía oral. A los 5 años requirió internación por neumonía, sin necesidad de suplemento de $\mathrm{O}_{2}$. Se indica la utilización de cough assist en forma diaria.

A los 5 años, se inició tratamiento con Nusinersen aplicado en forma intratecal, con el objetivo de estabilizar pautas motoras determinadas por la escala funcional de Hammersmith, que alcanzó valores de 13/66 en 2016. Luego de 10 meses del inicio del tratamiento demostró una mejoría motora que le permitió mejorar el sostén cefálico, mejorar la sedestación (Figura 2). Más aún, se observó un incremento de la escala de Hammersmith a 21/36.

A la fecha el niño se encuentra escolarizado con maestra integradora, realiza tratamiento kinésico regularmente y bajo tratamiento de mantenimiento con Nusinersen mostrando una favorable evolución.

\section{DISCUSIÓN}

Los avances en la identificación de genes relacionados con determinadas enfermedades neurológicas han contribuido al desarrollo de nuevas terapias; aunque la administración de los mismos continúa siendo un desafío. La progresión de esta enfermedad justifica la urgencia para prevenir enfermedades respiratorias que puedan traer complicaciones en la salud general del paciente, y en lo postural, evitar pérdida de funciones.

Nusinersen es un fármaco oligonucleotídico antisentido que modula el corte y empalme de ARN mensajero del gen de la neurona motora de supervivencia 2 (SMN2). Ha sido desarrollado para el tratamiento de la atrofia muscular espinal (AME) ${ }^{8}$.

Recientes estudios clínicos en fase 3 de tratamiento de Nusinersen mediante administración intratecal en niños con AME que presentaron síntomas después de los 6 meses de edad demostraron un significativo incremento en la puntuación Hammersmith Functional Motor Scale-Expanded (HFMSE) luego de 15 meses de tratamiento9. Los puntajes de HFMSE varían de 0 a 66. Los puntajes más altos indican una mejor función motora. Un incremento de más de 3 puntos de HFMSE en los niños tratados significa un aumento clínicamente significativo que indicaría mejoría en al menos dos habilidades motoras ${ }^{10}$. En el análisis final del estudio de Mercuri et al, concluyó que el 57\% de los niños en el grupo Nusinersen en comparación con el 26\% en el grupo control, tuvo un aumento desde el inicio hasta el mes 15 en la puntuación HFMSE de al menos 3 puntos $(p<0.001)$ y la incidencia general de eventos adversos fue similar en el grupo Nusinersen y el grupo control ( $93 \%$ y $100 \%$, respectivamente) $)^{9}$.

El niño presentado en este caso ha mostrado una buena evolución con las aplicaciones, mejorando a la fecha 8 puntos en la escala de HFMSE, sin ningún evento adverso. A pesar de estos resultados, el tratamiento es de muy difícil acceso para los niños con AME pese a los esfuerzos de la fundación de Familias de niños con AME $(\text { FAME })^{11}$. 


\section{BIBLIOGRAFÍA}

I. Kolb SJ, Kissel JT. Spinal Muscular Atrophy. Neurol Clin 20।5;33(4):83I-46.

2. Sugarman EA, Nagan $N$, Zhu H, et al. Pan-ethnic carrier screening and prenatal diagnosis for spinal muscular atrophy: clinical laboratory analysis of $>72,400$ specimens. Eur J Hum Genet 20 I2;20:27-32.

3. Lefebvre S, Burlet P, Liu Q, et al. Correlation between severity and SMN protein level in spinal muscular atrophy. Nat Genet 1997; 16(3):265-9.

4. Mailman MD, Heinz JW, Papp AC, et al. Molecular analysis of spinal muscular atrophy and modification of the phenotype by SMN2. Genet Med 2002; 4(I):20-6.

5. Kariyawasam D, Carey KA, Jones KJ, Farrar MA. New and developing therapies in spinal muscular atrophy. Paediatr Respir Rev 20 18. doi: | 0.10 |6/j.prrv.20 |8.03.003.
6. Finkel RS, Chiriboga CA,Vajsar J, et al.Treatment of infantile-onset spinal muscular atrophy with nusinersen:a phase 2, open-label, dose-escalation study. Lancet 20 I 6;388(I0063):30 I 7-26.

7. Finkel RS, Mercuri E, Darras BT, et al. Nusinersen versus Sham Control in Infantile-Onset Spinal Muscular Atrophy. N Engl J Med 2017;377( | 8): 1723-32.

8. Hoy SM. Nusinersen: First Global Approval. Drugs 20 17;77(4):473-79

9. Mercuri E, Darras BT, Chiriboga CA, et al. Nusinersen versus Sham Control in Later-Onset Spinal Muscular Atrophy. N Engl J Med 2018;15;378(7):625-35.

10. Pera MC, Coratti G, Forcina N. Content validity and clinical meaningfulness of the HFMSE in spinal muscular atrophy. BMC Neurol 2017;17(1):39

I।. Fundación Argentina de Familias con AME. www.fameargentina. com.ar, visitado el |8-07-20।8. 